\title{
ARTICLE OPEN Experimental detection of entanglement polytopes via local filters
}

\author{
Yuan-Yuan Zhao ${ }^{1,2}$, Markus Grassl ${ }^{3}$, Bei Zeng ${ }^{4,5}$, Guo-Yong Xiang ${ }^{1,2}$, Chao Zhang ${ }^{1,2}$, Chuan-Feng Li ${ }^{1,2}$ and Guang-Can Guo ${ }^{1,2}$
}

Quantum entanglement, resulting in correlations between subsystems that are stronger than any possible classical correlation, is one of the mysteries of quantum mechanics. Entanglement cannot be increased by any local operation, and for a sufficiently large many-body quantum system there exist infinitely many different entanglement classes, i.e., states that are not related by stochastic local operations and classical communications. On the other hand, the method of entanglement polytopes results in finitely many coarse-grained types of entanglement that can be detected by only measuring single-particle spectra. We find, however, that with high probability the local spectra lie in more than one polytope, hence providing only partial information about the entanglement type. To overcome this problem, we propose to additionally use so-called local filters, which are non-unitary local operations. We experimentally demonstrate the detection of entanglement polytopes in a four-qubit system. Using local filters we can distinguish the entanglement type of states with the same single particle spectra, but which belong to different polytopes.

npj Quantum Information (2017)3:11; doi:10.1038/s41534-017-0007-5

\section{INTRODUCTION}

Entanglement is an important resource in quantum information processing tasks, such as quantum teleportation, quantum errorcorrection, and quantum computing. ${ }^{1}$ Its amazing properties are very different from those of classical correlations, and many researches have been done to understand them. ${ }^{2}$ Essentially, entanglement comes from the tensor product structure of the Hilbert space of $N$ systems-qubits in our case. An $N$-qubit quantum state $\left|\Psi_{N}\right\rangle$ is entangled if it cannot be factored into products of quantum states of each of the qubits.

One central question regarding entanglement is that in which way $\left|\Psi_{N}\right\rangle$ may be entangled and how to detect that feature in practice. An obvious fact is that the number of parameters needed to specify $\left|\Psi_{N}\right\rangle$ grows exponentially with $N$. A natural idea to eliminate some of the free parameters is to consider two states $\left|\Psi_{N}\right\rangle$ and $\left|\Phi_{N}\right\rangle$ to have similar entanglement features if they can be connected by some single-qubit operations. For instance, if $\left|\Psi_{N}\right\rangle=$ $\otimes_{i=1}^{n} U_{i}\left|\Phi_{N}\right\rangle$ with each $U_{i}$ a unitary operation on qubit $i$, then $\left|\Psi_{N}\right\rangle$ and $\left|\Phi_{N}\right\rangle$ are said to be equivalent under local unitary (LU) transformation. $^{3-5}$ And if $\left|\Psi_{N}\right\rangle=\otimes_{i=1}^{n} M_{i}\left|\Phi_{N}\right\rangle$ with each $M_{i}$ an invertible operation on qubit $i$, then $\left|\Psi_{N}\right\rangle$ and $\left|\Phi_{N}\right\rangle$ are said to be equivalent under stochastic local operation and classical communication (SLOCC). ${ }^{6-8}$

Relative to LU and SLOCC transformations, many-body entanglement types can be classified up to local equivalence. That is, two LU/SLOCC equivalent states have similar entanglement properties. However, since the number of free parameters describing $U_{i}$ (or $M_{i}$ ) is linear in $N$, the number of entanglement classes still grows exponentially with $N$. This exponential growth of parameters for describing $\left|\Psi_{N}\right\rangle$ makes it hopeless to extract clear physical meanings of these classifications. It is, therefore, highly desired to coarse-grain these classes such that we can grasp the key features of each entanglement type. The concept of entanglement polytopes provides an elegant idea to meet this need. ${ }^{9}$ For each $N$, it results in only a finite number of coarse-grained entanglement classes, which we call entanglement types. More importantly, points in the polytope space can be detected directly in experiment via measuring only single-particle spectra of each qubit. ${ }^{9-11}$

To get a concrete idea about entanglement polytopes, we consider a system of $N$ qubits, and denote the single-particle reduced density matrix of qubit $i$ by $\rho_{i}$, where $i=1, \ldots, N$. For each $\rho_{i}$, there are two eigenvalues $\lambda_{i}^{a}$ and $\lambda_{i}^{\beta}$, with $\lambda_{i}^{a}+\lambda_{i}^{\beta}=1$. Keeping only the larger of the two eigenvalues of each reduced one-qubit density matrix $\rho_{i}$ (i.e., $\lambda_{i}^{\max }=\max \left\{\lambda_{i}^{a}, \lambda_{i}^{\beta}\right\}$ ), we obtain a vector $\vec{\lambda}=\left(\lambda_{1}^{\max }, \lambda_{2}^{\max }, \ldots, \lambda_{N}^{\max }\right)$ in the $N$-dimensional real space $\mathbb{R}^{N}$, which is the ambient space of the entanglement polytope. It turns out that the vectors $\vec{\lambda}$ associated with all pure states $\left|\Psi_{N}\right\rangle$ in the closure of an orbit under SLOCC transformations form a polytope. The vertices of those polytopes can be computed from so-called covariants that do not vanish identically on the orbit (for details see $^{9,11}$ ). Here, we only mention that the algebra of covariants is finitely generated, which implies that there are only finitely many vertices, and in turn finitely many different entanglement polytopes. In general, computing a generating set for the algebra of covariants and the entanglement polytopes is a non-trivial task.

For $N=2$, the Schmidt decomposition tells us that, up to LU, any pure state can be expressed as $\left|\Psi_{2}\right\rangle=\sqrt{\lambda_{1}^{\alpha}}|00\rangle+\sqrt{\lambda_{1}^{\beta}}|11\rangle$ with $\lambda_{1}^{a}+\lambda_{1}^{\beta}=1$. Moreover, the eigenvalues of $\rho_{2}$ are the same as that of $\rho_{1}$, i.e., $\lambda_{1}^{\alpha}=\lambda_{2}^{a}$ and $\lambda_{1}^{\beta}=\lambda_{2}^{\beta}$. Different values of $\lambda_{1}^{\max } \in[1 / 2,1]$ correspond to different LU classes of entanglement, which are in

\footnotetext{
${ }^{1}$ Key Laboratory of Quantum Information, University of Science and Technology of China, CAS, Hefei 230026, People's Republic of China; ${ }^{2}$ Synergetic Innovation Center of Quantum Information and Quantum Physics, University of Science and Technology of China, Anhui, Hefei 230026, People's Republic of China; ${ }^{3}$ Max-Planck-Institut für die Physik

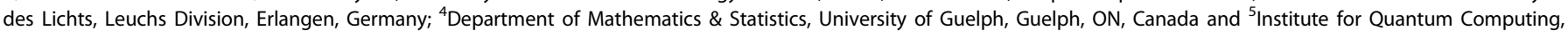
University of Waterloo, Waterloo, ON, Canada

Correspondence: Guo-Yong Xiang (gyxiang@ustc.edu.cn)
}

Received: 10 May 2016 Revised: 3 October 2016 Accepted: 15 December 2016

Published online: 20 March 2017 
fact infinitely many. Up to SLOCC, however, there is only one (nontrivial) class of entangled states, which contains the EPR pair with $\lambda_{1}^{\max }=1 / 2$. The entanglement polytope in this case is given by $\vec{\lambda}=\left(\lambda_{1}^{\max }, \lambda_{2}^{\max }\right)$, with the condition $\lambda_{1}^{\max }=\lambda_{2}^{\max } \in[1 / 2,1]$, i.e., a line connecting the points $(1 / 2,1 / 2)$ and $(1,1)$. The set of $\vec{\lambda}$ forms a polytope in $\mathbb{R}^{2}$, as illustrated in Fig. $1 \mathrm{a}$, and there is only one entanglement polytope for $N=2$.

For $N=3$, up to $\mathrm{LU}$, there are infinitely many equivalence classes that are described by 5 free parameters. ${ }^{3}$ Up to SLOCC, however, there are only two types of three-qubit-entanglement: the Greenberger-Horne-Zeilinger type (GHZ-type) and the $W$-type. ${ }^{6}$ These two types can be distinguished by a quantity called 3-tangle, ${ }^{12}$ which is, however, not a single-copy observable and hence cannot be directly measured in experiment (that is, to get the value of the 3-tangle, one either needs to measure jointly on multiple copies of the states, or needs to perform state tomography). ${ }^{13}$

In the $N=3$ case, the entanglement polytopes are given by the values of the vectors $\vec{\lambda}=\left(\lambda_{1}^{\max }, \lambda_{2}^{\max }, \lambda_{3}^{\max }\right)$. As illustrated in Fig. $1 \mathrm{~b}$, there are two polytopes: one corresponds to the $\mathrm{GHZ}$ class of entanglement, which is given by vertices $(1 / 2,1 / 2,1 / 2),(1,1 / 2$, $1 / 2),(1 / 2,1,1 / 2),(1 / 2,1 / 2,1),(1,1,1)$, and a smaller polytope inside that does not contain the vertex $(1 / 2,1 / 2,1 / 2)$ corresponds to the $\mathrm{W}$ class of entanglement.

For any $N=4$, SLOCC no longer results in a finite number of entanglement classes. In refs 7, 8 nine-different classes are obtained (with some classes containing free parameters resulting in fact in infinitely many-classes). However, there are only finite number of entanglement polytopes, whose vertices can be computed from those SLOCC classes and the covariants for four qubits (see ref. 9 for details). Consequently, unlike the $N=2$ and $N$ $=3$ case where the polytopes give essentially the same entanglement classes as the SLOCC classification, in general, entanglement polytopes coarse-grain the SLOCC classification (that is, different SLOCC classes may belong to the same entanglement polytope). Different entanglement polytopes form a nested hierarchy, ${ }^{9-11}$ which typically only allows a one-sided discrimination of entanglement types. Only if the local spectra of $\left|\Psi_{N}\right\rangle$ happen to lie in a non-overlapping region, one can tell for certain which polytope $\left|\Psi_{N}\right\rangle$ belongs to. As an example, in the $N=3$ case, the $\mathrm{W}$ class polytope sits inside the GHZ class polytope (as illustrated in Fig. $1 \mathrm{~b}$, hence only if the local spectra of some state lie outside the W class polytope one can tell that the state has GHZ-type entanglement. This means that a point $\vec{\lambda} \in \mathcal{P}^{W}$ fails to distinguish $W$-type entanglement from GHZ-type entanglement. A recent experiment has demonstrated the detection of entanglement polytopes by measuring local spectra, where the states have been chosen such that their local spectra lie in non-overlapping regions. ${ }^{14}$ For a general use of the entanglement polytope method, however, it is crucial to understand the relation between the different polytopes and how large their overlaps can be.

In this work, we show that, unfortunately, the overlap is considerably large. That is, in general, for a randomly chosen $\mathrm{N}$ qubit state $\left|\Psi_{N}\right\rangle$, with high probability the vector of local spectra falls in some region of overlapping polytopes. For instance, in the $N=3$ case, for a uniformly random pure three-qubit state with respect to the Haar measure, the resulting joint distribution of the eigenvalues of the local density matrices has been computed in ref. 15. From this results, one finds that the volume of the subpolytope $\mathcal{P}^{W}$ with respect to the Haar measure is $203 / 216 \approx 93.98 \%$, while its geometric volume is $1 / 3$ of the full polytope (see Fig. 1b). Hence the probability for a random three-qubit state to have a vector of local spectra corresponding to a point outside the polytope $\mathcal{P}^{W}$ is only $13 / 216 \approx 6.02 \%$.

To overcome this difficulty, we propose to additionally use SLOCC operations. That is, if the local spectra of $\left|\Psi_{N}\right\rangle$ lies in an overlapping region, then after applying an SLOCC operation $L$, the

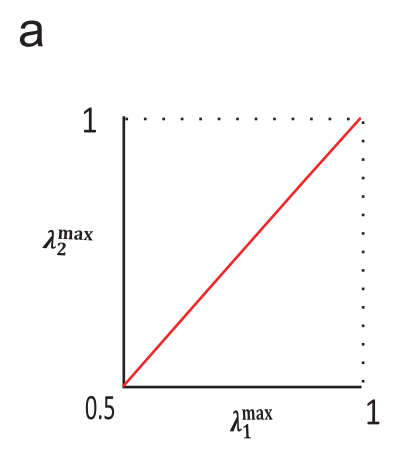

b

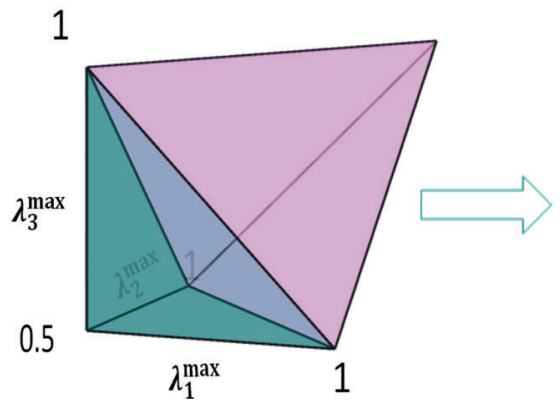

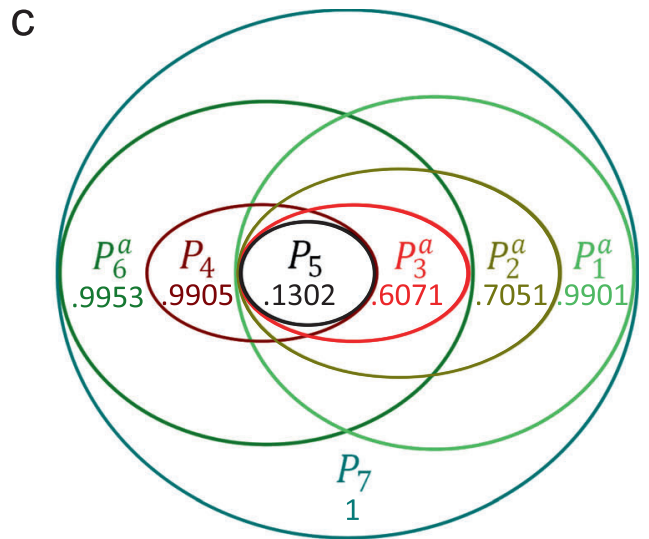

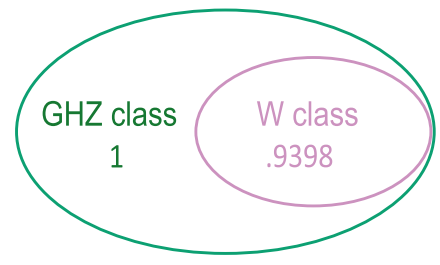

Fig. $1 N$-qubit entanglement polytopes for $N=2,3,4$. The coordinates $\lambda_{i}^{\max }$ in $\mathbf{a}$ and $\mathbf{b}$ are given by the lager of the two eigenvalues of the single-particle reduced density matrix of qubit $i$. a Two-qubit entanglement polytope (the red line). $\mathbf{b}$ Three-qubit entanglement polytopes. The pink part represents the W-class, and the probability of a random state lying in this region is about 0.9398 . c Nested structure of the different types of four-qubit entanglement polytopes. Together with the label $\mathcal{P}_{i}^{m}$ for the various types of polytopes, we give the probability that a random pure state lies in the that polytope (sampling $10^{6}$ states, see Supplementary Material, Table 2) 
local spectra of the state $L\left|\Psi_{N}\right\rangle$ has some chance to fall into a nonoverlapping region. If not, we repeat this procedure again with a different SLOCC operation. After several rounds with different SLOCC operations, we either find a point that lies in a nonoverlapping region, or we assume that the state in fact lies in the smaller polytope of the hierarchy.

We experimentally demonstrate the detection of entanglement polytopes in a four-qubit optical system. We use the technique of local filters (see, e.g., refs 16-21) to implement SLOCC operations. For the states we tested, after several rounds, the local filters allow to effectively distinguish states with the same single particle spectra, but which belong to different polytopes. Our method provides an effective way of detecting the entanglement type based on only local filters and local spectra, which may shed light on the techniques of entanglement detection in more general settings.

\section{RESULTS}

Four-qubit polytopes

In the case of four-qubit pure states, there are infinitely many SLOCC orbits, but only finitely many entanglement polytopes. The full polytope, containing $\vec{\lambda}$ for any four-qubit state, denoted by $\mathcal{P}^{\text {full }}$, is spanned by the vertices (see ref. 9)

$$
\begin{array}{r}
\left(\frac{1}{2}, \frac{1}{2}, \frac{1}{2}, \frac{1}{2}\right), \\
\left(1, \frac{1}{2}, \frac{1}{2}, \frac{1}{2}\right),\left(\frac{1}{2}, 1, \frac{1}{2}, \frac{1}{2}\right),\left(\frac{1}{2}, \frac{1}{2}, 1, \frac{1}{2}\right),\left(\frac{1}{2}, \frac{1}{2}, \frac{1}{2}, 1\right), \\
\left(1,1, \frac{1}{2}, \frac{1}{2}\right),\left(1, \frac{1}{2}, 1, \frac{1}{2}\right),\left(1, \frac{1}{2}, \frac{1}{2}, 1\right),\left(\frac{1}{2}, 1,1, \frac{1}{2}\right),\left(\frac{1}{2}, 1, \frac{1}{2}, 1\right),\left(\frac{1}{2}, \frac{1}{2}, 1,1\right),
\end{array}
$$

Up to permutation of the qubits, there are six other polytopes inside $\mathcal{P}^{\text {full }}$, which may also be mutually overlapping. A full list of all these polytopes and their relationship can be found in Section $\mathrm{B}$ of the supplementary material. The polytopes are labeled by $\mathcal{P}_{i}^{m}$, where the index $i=1, \ldots, 7$ denotes the seven different types of polytopes (following the naming convention of ref. 9), and the superscript $m=a, b, \ldots$ labels the polytopes that differ only by a permutation of vertices, corresponding to permutations of the qubits. We computed the local spectra of $10^{6}$ random pure states and determined which of the polytopes contains the vector of local spectra. The results are summarized in Fig. 1c. For each of the seven types of polytopes, only one representative $\mathcal{P}_{i}^{a}$ is shown when the polytope is no invariant under permutations. Our results demonstrate that, similar as in the $N=3$ case, for a randomly chosen pure four-qubit state, the chance that $\vec{\lambda}$ lies in an overlapping region is high. Therefore, we have to apply local operations to 'move' $\vec{\lambda}$.

\section{Experiment protocol}

In our experiment, two different four-qubit states $\left|\Psi^{(1)}\right\rangle$ and $\left|\Psi^{(2)}\right\rangle$ are prepared, where

$$
\left|\Psi^{(1)}\right\rangle=\frac{\sqrt{3}}{3}(|H H H H\rangle+|V V V\rangle)+\frac{\sqrt{3}}{6}(|H V\rangle+|V H\rangle)(|H V\rangle+|V H\rangle),
$$

and $\left|\Psi^{(2)}\right\rangle$ is the four-qubit $\mathrm{GHZ}$ state

$$
\left|\Psi^{(2)}\right\rangle=\frac{\sqrt{2}}{2}(|H H H H\rangle+|V V V V\rangle) \text {. }
$$

The qubits are encoded by horizontal $|H\rangle$ and vertical $|V\rangle$ polarization. The goal is to determine the entanglement type for each of the input states using the entanglement polytope method. For both $\left|\psi^{(1)}\right\rangle$ and $\left|\psi^{(2)}\right\rangle$, we have $\vec{\lambda}=\left(\frac{1}{2}, \frac{1}{2}, \frac{1}{2}, \frac{1}{2}\right)$, that is, the local spectra do not tell the states apart. Hence local filter operations are needed to 'move' $\vec{\lambda}$.

The closure of the SLOCC orbit of the four-qubit GHZ state $\left|\Psi^{(2)}\right\rangle$ corresponds to the full polytope $\mathcal{P}^{\text {full }}=\mathcal{P}_{7}$. However, the state $\left|\Psi^{(1)}\right\rangle$ corresponds to a smaller polytope $\mathcal{P}^{s}=\mathcal{P}_{4} \subset \mathcal{P}^{\text {full }}$ with vertices

$$
\left(1,1, \frac{1}{2}, \frac{1}{2}\right),\left(1, \frac{1}{2}, 1, \frac{1}{2}\right),\left(1, \frac{1}{2}, \frac{1}{2}, 1\right),\left(\frac{1}{2}, 1,1, \frac{1}{2}, \frac{1}{2}, \frac{1}{2}\right),\left(\frac{1}{2}, 1, \frac{1}{2}, 1\right),\left(\frac{1}{2}, \frac{1}{2}, 1,1\right),
$$

The smaller polytope $\mathcal{P}^{s}=\mathcal{P}_{4}$ is separated from the full polytope $\mathcal{P}^{\text {full }}$ by the additional constraint

$f_{1}(\vec{\lambda})=-\lambda_{1}^{\max }+\lambda_{2}^{\max }+\lambda_{3}^{\max }+\lambda_{4}^{\max } \geq 1$

and all permutations of it. Combining those four inequalities, we have the equivalent conditions

$$
\begin{aligned}
& f(\vec{\lambda}):=\min _{i=1,2,3,4} f_{i}(\vec{\lambda}) \geq 1 \\
& \min _{i=1,2,3,4}\left(\sum_{j=1}^{4} \lambda_{j}^{\max }\right)-\lambda_{i}^{\max } \geq 1 \\
& \left(\sum_{j=1}^{4} \lambda_{j}^{\max }\right)-\max _{i=1,2,3,4} \lambda_{i}^{\max } \geq 1
\end{aligned}
$$

A state lies outside of the smaller polytope when the left hand side $f(\vec{\lambda})$ of those conditions is strictly smaller than 1 .

While the polytope $\mathcal{P}_{4}$ corresponding to the state $\left|\Psi^{(1)}\right\rangle$ of our experiment is fairly low in the hierarchy of polytopes (see Fig. 1c), the local spectra of only 9522 out of one million random states violate the discriminating inequalities (4). Hence, the chance for a random four-qubit state to have local spectra that lie outside of $\mathcal{P}_{4}$ is only about $0.95 \%$. This clearly indicates that one has to apply local filters in order to have a chance to get information about the entanglement polytopes.

Note that after measuring the first qubit of the state and postselection on the measurement outcome, we have a four-qubit state that factors into a single qubit and a three-qubit state. The first component $\lambda_{1}^{\max }=1$ is fixed, and we can map the polytope $\mathcal{P}_{4}$ to a three-qubit polytope. From the vertices in (3) we select those with $\lambda_{1}^{\max }=1$ and project onto the last three coordinates. Thereby we obtain the four vertices

$$
(1,1,1),(1,1 / 2,1 / 2),(1 / 2,1,1 / 2),(1 / 2,1 / 2,1)
$$

spanning the three-qubit $W$-polytope $\mathcal{P}^{W}$, which has a volume of about $94 \%$. Hence the local measurement and post-selection increase the chance for a random state to lie outside the smaller polytope from less than one percent to about six percent. As $\lambda_{1}^{\max }=1$ achieves the maximum value, the separating conditions reduce to the single condition

$f(\vec{\lambda})=\lambda_{2}^{\max }+\lambda_{3}^{\max }+\lambda_{4}^{\max }-1 \geq 1$.

Set-up

Our experiment demonstrates the preparation and detection of entanglement types of four-qubit states. We use non-unitary SLOCC operations, so-called local filters, ${ }^{16}$ to move $\vec{\lambda}$. (Note that these operations are different from, e.g., narrow-band frequency filters for photons used in experimental set-ups.) The proposed experiment is given by the diagram in Fig. 2 . 
Here $\vartheta_{i}$ for $i=1,2$ denotes a unitary local transformation $U_{\vartheta_{i}}$ of the form

$$
\mathrm{U}_{\vartheta_{i}}=\left(\begin{array}{cc}
\cos \vartheta_{i} & -\sin \vartheta_{i} \\
\sin \vartheta_{i} & \cos \vartheta_{i}
\end{array}\right)\left(\begin{array}{rr}
1 & 0 \\
0 & -1
\end{array}\right)\left(\begin{array}{cc}
\cos \vartheta_{i} & \sin \vartheta_{i} \\
-\sin \vartheta_{i} & \cos \vartheta_{i}
\end{array}\right) \text {, }
$$

and $\gamma$ denotes a non-unitary local transformation

$A_{\gamma}=\left(\begin{array}{ll}1 & 0 \\ 0 & \gamma\end{array}\right)$

In Fig. 2, two of the qubits encounter non-unitary local transformations: qubit 1 is measured in some basis and postselected, corresponding to the limit $\gamma \rightarrow 0$ for $A_{\gamma}$ and resulting in $\lambda_{1}^{\max }=1$; qubit 4 is going through a filter operation given by $A_{\gamma}$. In the most general case, one can also apply local filter operations (or measurements) on the other qubits. However, a single filter (or measurement) may already suffice to 'move' $\vec{\lambda}$ to non-overlapping regions of the polytopes, depending on the input state $\left|\boldsymbol{\Psi}^{(i)}\right\rangle$.

\section{Experimental results}

We first perform tomography on each single qubit to obtain the corresponding single-qubit density matrices and calculate their local spectra for both states. As shown in Table 1a, the local

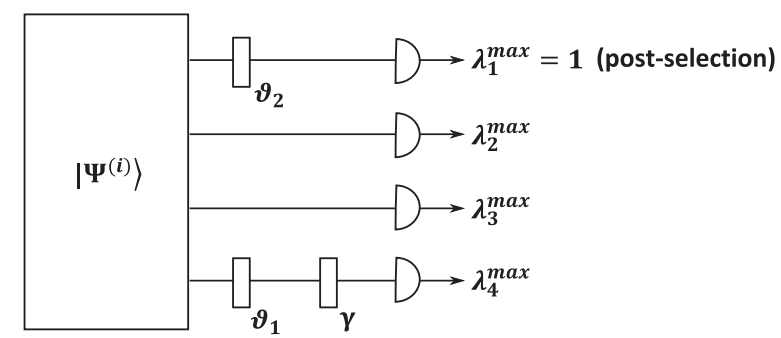

Fig. 2 Circuit diagram of the experimental set-up. The box labeled $\left|\Psi^{(i)}\right\rangle(i=1,2)$ stands for the preparation of the corresponding input state. The elements with the label $\vartheta_{i}(i=1,2)$ denote local unitary transformation $U_{\vartheta_{i}}$ on qubit 1 and 4. The element labeled $\gamma$ corresponds to the non-unitary transformation $A_{\gamma}$ (detailed forms can be found in Section Set-up). At the end, qubit 1 is measured in the computational basis, and only cases with the outcome $|0\rangle$ are considered (post-selection). For the other three qubits, local tomography yields the maximal eigenvalue $\lambda_{i}^{\max }$ spectra of $\left|\boldsymbol{\Psi}^{(1)}\right\rangle$ and $\left|\boldsymbol{\Psi}^{(2)}\right\rangle$ are almost identical, so we cannot distinguish their entanglement polytopes.

To distinguish the entanglement polytopes of $\left|\boldsymbol{\Psi}^{(1)}\right\rangle$ and $\left|\boldsymbol{\Psi}^{(2)}\right\rangle$, we try to move $\vec{\lambda}$ out of the smaller polytope $\mathcal{P}^{s}$ using local filters, as illustrated in Fig. 2. We fix $\vartheta_{2}=-\pi / 8$, and then measure the first qubit in the computational basis. By post-selection, we have $\lambda_{1}^{\max }=1$. For each setting of $\vartheta_{1}$ and $\gamma$, we perform tomography of the qubits 2,3 , and 4 to determine the values of $\lambda_{2}^{\max }, \lambda_{3}^{\max }$, and $\lambda_{4}^{\max }$ (see Table $1 \mathrm{~b}$ and Supplementary Material, Table 3 ). The smaller polytope $\mathcal{P}^{s}$ is characterized by $f(\vec{\lambda}) \geq 1$.

The results are illustrated in Fig. 3 . The data is shown in the three-dimensional polytope for $\lambda_{2}^{\max }, \lambda_{3}^{\max }, \lambda_{4}^{\max }$ as by post-selection of the first qubit, $\lambda_{1}^{\max }=1$. As discussed above, the smaller polytope $\mathcal{P}^{s}$ is mapped to the three-qubit polytope $\mathcal{P}^{W}$, while the image of the full polytope $\mathcal{P}^{\text {full }}$ has an additional vertex $(1 / 2,1 / 2$, $1 / 2)$. The data point $f$ of the state $\left|\boldsymbol{\Psi}^{(2)}\right\rangle$ outside of $\mathcal{P}^{W}$ shows that $\left|\boldsymbol{\Psi}^{(2)}\right\rangle$ is not in $\mathcal{P}^{s}$. In contrast, the data points $a, b, c, d, e$ obtained from $\left|\boldsymbol{\Psi}^{(1)}\right\rangle$ all lie in $\mathcal{P}^{W}$, which indicates that $\left\langle\boldsymbol{\Psi}^{(1)}\right\rangle$ belongs to $\mathcal{P}^{s}$. This shows that $\left|\boldsymbol{\Psi}^{(1)}\right\rangle$ and $\left|\boldsymbol{\Psi}^{(2)}\right\rangle$ have different entanglement types. To further support the assumption that $\left|\Psi^{(1)}\right\rangle$ belongs to $\mathcal{P}^{s}$, we obtain more data points for $\left|\boldsymbol{\Psi}^{(1)}\right\rangle$, using different local filters. The results are shown as dark dots in Fig. 3.

In Fig. 4, the original spectra of the four-qubit state $\left|\boldsymbol{\Psi}^{(1)}\right\rangle$ and $\left|\boldsymbol{\Psi}^{(2)}\right\rangle$ are plotted with red and blue circles, respectively. The horizontal axis corresponds to $\lambda_{4}^{\max }$, while the vertical axes gives the value of $-\lambda_{1}^{\max }+\lambda_{2}^{\max }+\lambda_{3}^{\max }$. We can see that the two local spectra are at almost the same position outside the shaded region, and we cannot distinguish their entanglement polytopes. Using local filters to move $\vec{\lambda}$, the results are plotted with dots in different colors. The blue dot in the cyan region violates the constraint (4). This implies that the state $\left|\boldsymbol{\Psi}^{(2)}\right\rangle$ is not in $\mathcal{P}^{s}$. In contrast, all the red dots obtained for $\left|\boldsymbol{\Psi}^{(1)}\right\rangle$ satisfy $f(\vec{\lambda}) \geq 1$, which indicates that $\left|\boldsymbol{\Psi}^{(1)}\right\rangle$ belongs to $\mathcal{P}^{s}$.

Since the first photon of the four-qubit state is post-selected and the last photon goes through a non-unitary filter, there is a finite probability to obtain a data point: for example, the probability of success is $0.153,0.231,0.205,0.279,0.5$, and 0.5 for our experimental data ' $a \sim f$,' respectively.

\section{Experimental error analysis}

Since the birefringence of the ordinary and extra-ordinary light (olight and $e$-light) in the down-converter creating a photon pair (BBO crystal) cannot be compensated completely, because of the

Table 1. Local spectra of the original four qubit states and the spectra after applying local operations

\begin{tabular}{|c|c|c|c|c|c|}
\hline \multicolumn{6}{|c|}{ (a) The local spectra $\lambda_{1}^{\max }, \lambda_{2}^{\max }, \lambda_{3}^{\max }, \lambda_{4}^{\max }$ together with $f(\vec{\lambda})$ for the states $\left|\boldsymbol{\Psi}^{(1)}\right\rangle$ and $\left|\boldsymbol{\Psi}^{(2)}\right\rangle$} \\
\hline State & $\lambda_{1}^{\max }$ & $\lambda_{2}^{\max }$ & $\lambda_{3}^{\max }$ & $\lambda_{4}^{\max }$ & $f(\vec{\lambda})$ \\
\hline$\left|\Psi^{(1)}\right\rangle$ & $0.549(4)$ & $0.540(4)$ & $0.532(4)$ & $0.531(4)$ & $1.054(8)$ \\
\hline$\left|\boldsymbol{\Psi}^{(2)}\right\rangle$ & $0.529(4)$ & $0.515(4)$ & $0.546(4)$ & $0.522(4)$ & $1.020(8)$ \\
\hline
\end{tabular}

(b) Setting of the parameters $\vartheta_{1}$ ('none' means that HWP2 was removed) and $\gamma$ for the data points labeled ' $a \sim f$, together with the measured local spectra $\lambda_{2}^{\max }, \lambda_{3}^{\max }, \lambda_{4}^{\max }$, as well as the resulting value of $f(\vec{\lambda})$

\begin{tabular}{lllllll}
\hline & $\vartheta_{1}$ & $\gamma$ & $\lambda_{2}^{\max }$ & $\lambda_{3}^{\max }$ & $\lambda_{4}^{\max }$ & $f(\vec{\lambda})$ \\
\hline $\mathrm{a}$ & $\pi / 8$ & $1 \sqrt{6}$ & $0.634(8)$ & $0.887(8)$ & $0.566(8)$ & $0.665(8)$ \\
$\mathrm{b}$ & $3 \pi / 32$ & $1 \sqrt{3}$ & $0.619(9)$ & $0.852(8)$ & $0.087(15)$ & $1.137(15)$ \\
$\mathrm{c}$ & 0.44 & $1 \sqrt{3.5}$ & $0.549(9)$ & $0.869(8)$ & $0.610(9)$ & $1.027(16)$ \\
$\mathrm{d}$ & $3 \pi / 16$ & $1 \sqrt{2.5}$ & $0.652(9)$ & $0.834(9)$ & $0.755(9)$ & $1.241(16)$ \\
$\mathrm{e}$ & None & 1 & $0.659(10)$ & $0.544(10)$ & $0.523(9)$ & $0.584(8)$ \\
$\mathrm{f}$ & None & 1 & $0.525(9)$ &
\end{tabular}

The uncertainties inside the brackets are obtained by Monte Carlo simulation (1000 runs) 


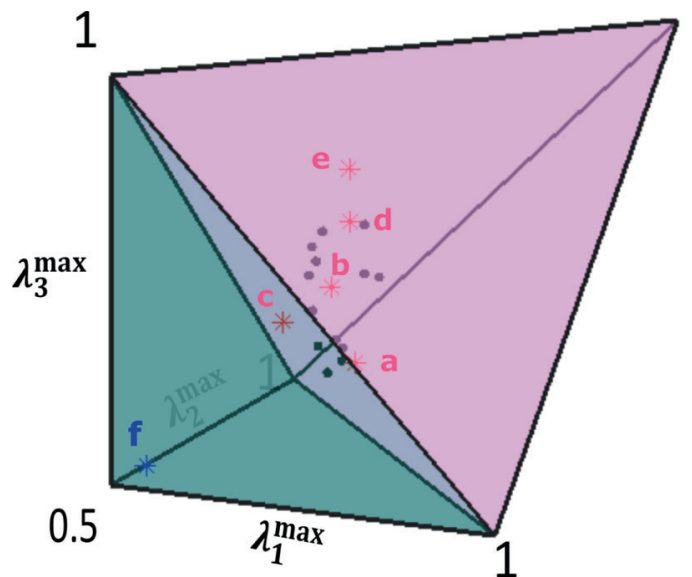

Fig. 3 Three-dimensional polytopes. The pink region represents the $\mathrm{W}$ class, and the union of the pink region and the green region is the $\mathrm{GHZ}$ class. The red stars and the dark dots are for the state $\left|\boldsymbol{\Psi}^{(1)}\right\rangle$ while the blue star ' $f$ ' is for the state $\left|\Psi^{(2)}\right\rangle$. Detailed values for the data points $a, b, c, d, e$, and $f$ can be found in Table $1 \mathrm{~b}$; for the rest see Supplementary Material, Table 3

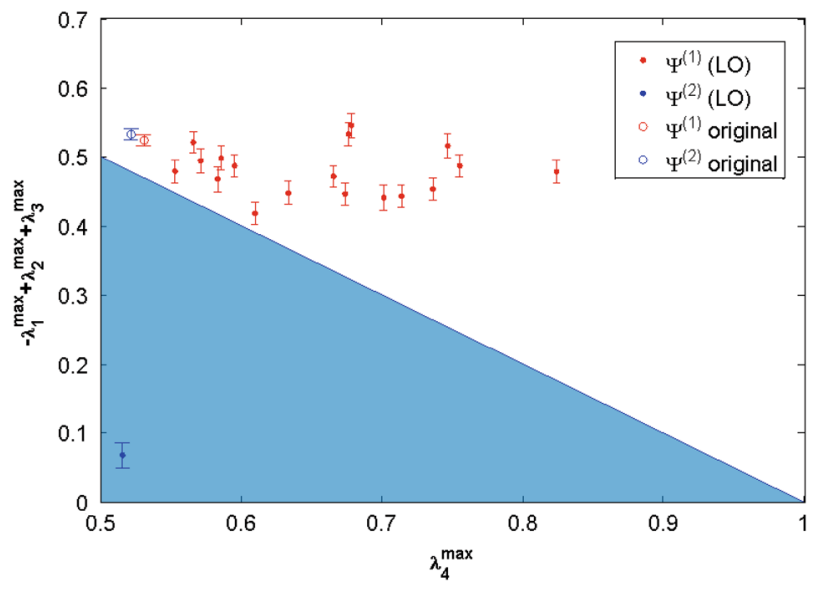

Fig. 4 Experimental results. The red and the blue circle are the local spectra of the original four qubit states. After applying local operations, the spectra are shown as the red and blue dots for $\left|\Psi^{(1)}\right\rangle$ and $\left|\Psi^{(2)}\right\rangle$, respectively. The data points in the cyan region do not meet the constraint (4). Error bars are obtained by Monte Carlo simulation (1000 runs)

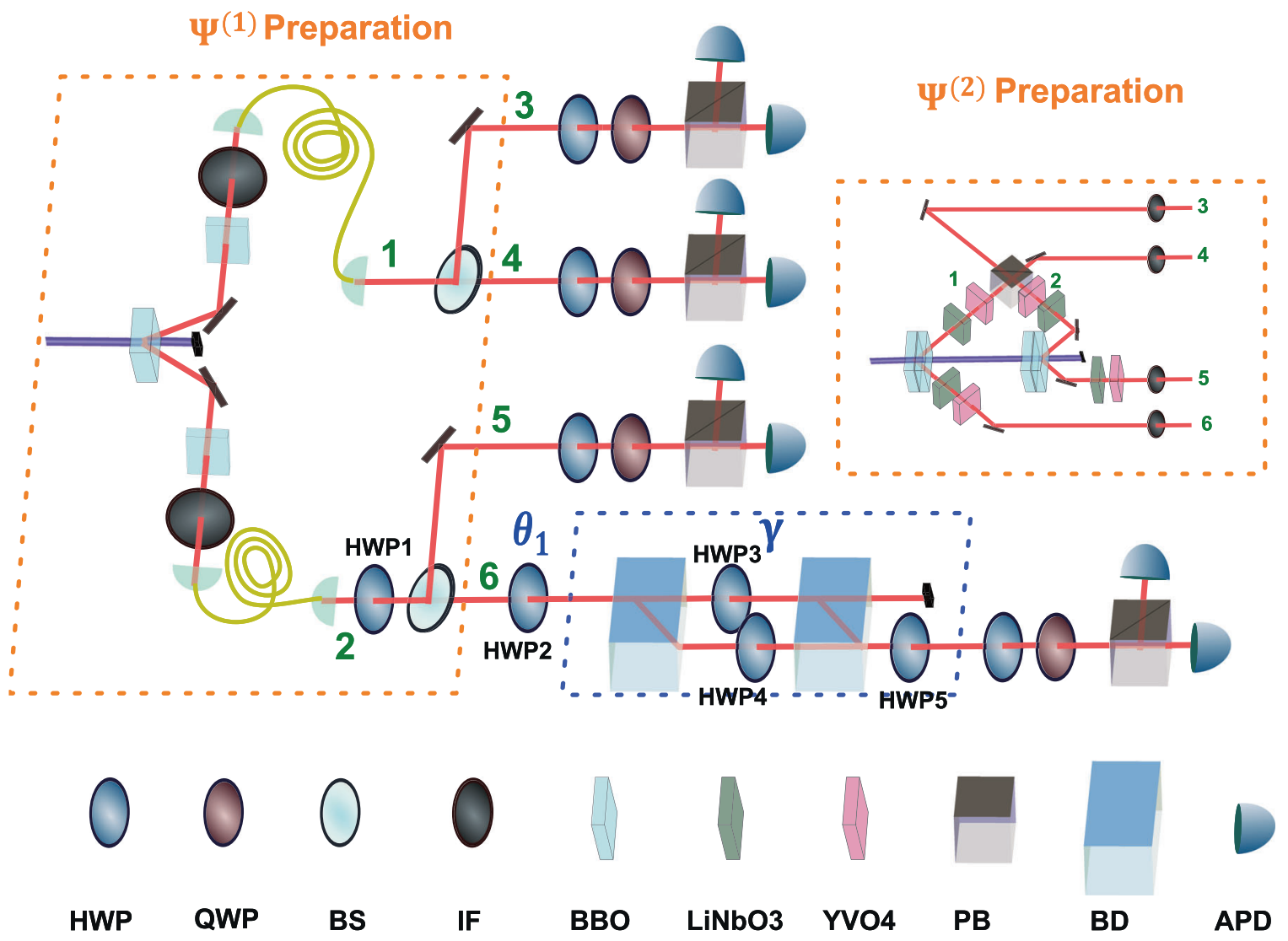

Fig. 5 Experimental set-up. Detailed configurations for preparing the states $\left|\Psi^{(1)}\right\rangle$ and $\left|\Psi^{(2)}\right\rangle$ and for realizing the operator $A_{\gamma}$ are shown in the corresponding boxes. The unitary operator $\mathrm{U}_{\vartheta}$ is realized by HWP2 at specific angles. A quarter-wave plate (QWP) and a half-wave plate (HWP) in front of a polarization beam splitter (PBS) in each mode are used to implement the measurement in different bases for the standard state tomography. All the photons are detected by avalanche photo-diodes (APD). Post-selection in some basis of qubit 1 (the state of the photon in mode ' 3 ') is realized by collecting only photons in one of the output modes of the PBS. The indices in the figure denote the spatial modes

high-term noise from the spontaneous parametric downconversion process, and some mode mismatch, we do not obtain the pure states $\left|\boldsymbol{\Psi}^{(1)}\right\rangle$ and $\left|\boldsymbol{\Psi}^{(2)}\right\rangle$, but some noisy version of them. The errors of the local filters are mainly due to non-perfect beam alignment and angle-settings of half-wave plates (HWPs).

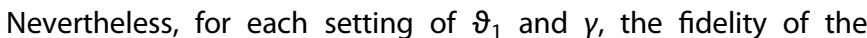
post-selected three-qubit state is over 0.90 . The coincidence counts of the photon pairs generated in the BBO obey a Poisson distribution, the parameters of which we estimate from the experimental data. We use Monte Carlo simulation to estimate the 
errors of the results. Firstly, we generate 1000 groups of random numbers from the Poisson distribution with the original experimental data as mean parameters, then we calculate the corresponding spectra for each group of generated data and obtain the standard deviation.

\section{DISCUSSION}

The experimental data points clearly show that $\left|\Psi^{(2)}\right\rangle$ is outside of the smaller polytope, while for the state $\left|\boldsymbol{\Psi}^{(1)}\right\rangle$ they indicate that it lies inside the smaller polytope, i.e., the two pure states have different types of entanglement. Clearly, a single point outside the pink region in Fig. 3 suffices to show that $\left|\boldsymbol{\Psi}^{(2)}\right\rangle$ does not belong to $\mathcal{P}^{s}$. The five data points in the pink region for the state $\left|\Psi^{(1)}\right\rangle$ suggest that it belongs to $\mathcal{P}^{s}$. Recall that the criteria based on entanglement polytopes are in general only one-sided, and in order to get a conclusive answer about the entanglement type for all states, one has to perform full state tomography. ${ }^{22}$

We perform a full state tomography to reconstruct the density matrix of $\left|\boldsymbol{\Psi}^{(1)}\right\rangle$ and $\left|\boldsymbol{\Psi}^{(2)}\right\rangle$ with Pauli measurements and using an efficient linear regression estimation algorithm. ${ }^{23}$ The fidelities of the states in the experiment are $0.951 \pm 0.004$ and $0.900 \pm 0.004$, respectively. In Supplementary Material, Fig. 2, we compare the ideal state with the state prepared in the experiment.

In summary, we experimentally demonstrate the detection of entanglement polytopes in a four-qubit system. We use local filters to effectively distinguish states with the same single-particle spectra, but which belong to different polytopes. This provides a new tool to experimental detection of entanglement in a multiqubit system using only local operations.

\section{METHODS}

Experimental state preparation

Our experimental set-up for the preparation of the states $\left|\Psi^{(1)}\right\rangle$ and $\left|\Psi^{(2)}\right\rangle$ is shown in Fig. 5. A $390 \mathrm{~nm}$ femto-second pump light, frequency-doubled from a $780 \mathrm{~nm}$ mode-locked Ti:sapphire pulsed laser (with a pulse width of about $150 \mathrm{fs}$ and repetition rate $76 \mathrm{MHz}$ ), is used to pump the respective down-converter. For the preparation of $\left|\boldsymbol{\Psi}^{(1)}\right\rangle$, a $2 \mathrm{~mm}$ type-II phasematched BBO crystal is used as down-converter to produce two pairs of entangled photons, ${ }^{24} \frac{1}{2}(|H V\rangle+|V H\rangle) \otimes(|H V\rangle+|V H\rangle)$, and two $1 \mathrm{~mm} \mathrm{BBO}$ crystals are used to compensate the birefringence of $o$-light and $e$-light in the $2 \mathrm{~mm} \mathrm{BBO}$. HWP1 rotates the polarization of the photons in path ' 2 ' (horizontal to vertical and vertical to horizontal). After the beam splitters, we post-select the case that each outport has only one photon, then the above two pairs of entangled photons are transformed into the state $\left|\boldsymbol{\Psi}^{(1)}\right\rangle$. For the four-qubit $\mathrm{GHZ}$ state $\left|\boldsymbol{\Psi}^{(2)}\right\rangle$ shown in the right part of Fig. 5, a cascaded sandwich beam-like $\mathrm{BBO}$ entangled source ${ }^{25}$ is used, where the $\mathrm{LiNbO}_{3}$ crystals are for spatial compensations and the $\mathrm{YVO}_{4}$ crystals are for temporal compensations. The ultra-fast pump beam passes through the two sandwich BBO crystals to generate two pairs of entangled photons, $\frac{1}{2}(|H V\rangle+|V H\rangle) \otimes(|H V\rangle+|V H\rangle)$, and a polarizing beam splitter (PBS) combines the photons from modes ' 1 ' and ' 2 ' through a Hong-OuMandel interferometer. The PBS acts as a parity check gate $|H H\rangle\langle H H|+$ $|V V\rangle\langle V V|$ when we collect the two photons from two different outports. We will get the four-qubit GHZ state $\left|\boldsymbol{\Psi}^{(2)}\right\rangle$ if there is one photon in each of the modes ' 3 ', '4', '5', and '6' (ref. 26).

\section{Local filter}

We use two beam displacers (BD) and three half-wave plates (HWP3, HWP4, and HWP5) to construct the local filter operations $A_{\gamma}$. The three HWPs are rotated by $\frac{1}{2}(\arcsin \gamma), \frac{\pi}{4}$, and $\frac{\pi}{4}$, respectively. The designed BD we use does not displace the vertically polarized photons, but makes the horizontally polarized ones undergo a $4 \mathrm{~mm}$ lateral displacement. The $\frac{\pi}{4}$ HWPs are used to change the polarization from $\mathrm{H}$ to $\mathrm{V}$ (from $\mathrm{V}$ to $\mathrm{H}$ ). For the horizontally polarized photons, they all pass through BD1, HWP4, and $\mathrm{BD} 2$, and then they are collected by the fibers. However, for the vertically polarized photons, after BD1 and HWP3, only a part of them undergoes the lateral displacement and is collected. To ensure that the photons from different paths of BD2 are all collected by the multimode fibers, we adjust a Mach-Zehnder type interferometer whose visibility is about $98 \%$.

\section{ACKNOWLEDGEMENTS}

M.G., G.Y.X., and B.Z. would like to thank the Fields Institute, Toronto, for hospitality during the 2013 Workshop on Mathematical Methods of Quantum Tomography, where this work was initiated. The work at USTC is supported by National Natural Science Foundation of China (Grants No. 11574291, No. 61108009 and No. 61222504). B.Z. is supported by NSERC and CIFAR.

\section{AUTHOR CONTRIBUTIONS}

B.Z. and M.G. developed the theoretical model; G.Y.X. designed the experiment; Y.Y.Z. performed the experiment with the help of C.Z. and G.Y.X.; B.Z., M.G., and Y.Y.Z. analyzed the data and wrote the manuscript with the help from G.Y.X., C.F.L., and G.C. G.; all authors read the paper and discussed the results.

\section{COMPETING INTERESTS}

The authors declare no competing financial interests.

\section{REFERENCES}

1 Nielsen, M. A. \& Isaac L. C.Quantum Computation and Quantum Information (Cambridge Univ. Press, 2010).

2 Horodecki, R., Horodecki, P., Horodecki, M. \& Horodecki, K. Quantum entanglement. Rev. Mod. Phys. 81, 865-942 (2009).

3 Acin, A., Andrianov, A., Jané, E. \& Tarrach, R. Three-qubit pure-state canonical forms. J. Phys. A: Math. Gen 34, 6725-6739 (2001).

4 Chen, L., oković, D. Ž., Grassl, M. \& Zeng, B. Canonical form of three-fermion purestates with six single particle states. J. Math. Phys 55, 082203 (2014).

5 Chen, L., Chen, J., oković, D. Ž. \& Zeng, B. Universal subspaces for local unitary groups of fermionic systems. Commun. Math. Phys. 333, 541-563 (2015).

6 Dür, W., Vidal, G. \& Cirac, J. I. Three qubits can be entangled in two inequivalent ways. Phys. Rev. A. 62, 062314 (2000).

7 Verstraete, F., Dehaene, J., De Moor, B. \& Verschelde, H. Four qubits can be entangled in nine different ways. Phys. Rev. A. 65, 052112 (2002).

8 Chterental, O.\&oković, D. Ž., Normal forms and tensor ranks of pure states of four qubits. arXiv preprint quant-ph/0612184 (2006).

9 Walter, M., Doran, B., Gross, D. \& Christandl, M. Entanglement polytopes: multiparticle entanglement from single-particle information. Science 340, 1205-1208 (2013).

10 Sawicki, A., Oszmaniec, M. \& Kuś, M. Critical sets of the total variance can detect all stochastic local operations and classical communication classes of multiparticle entanglement. Phys. Rev. A. 86, 040304 (2012).

11 Sawicki, A., Oszmaniec, M. \& Kuś, M. Convexity of momentum map, morse index, and quantum entanglement. Rev. Math. Phys. 26, 1450004 (2014).

12 Coffman, V., Kundu, J. \& Wootters, W. K. Distributed entanglement. Phys. Rev. A. 61, 052306 (2000).

13 Gühne, O. \& Tóth, G. Entanglement detection. Phys. Rep. 474, 1-75 (2009).

14 Aguilar, G. H., Walborn, S. P., Souto Ribeiro, P. H. \& Céleri, L. C. Experimental determination of multipartite entanglement with incomplete information. Phys. Rev. X 5, 031042 (2015).

15 Christandl, M., Doran, B., Kousidi, S. \& Walter, M. Eigenvalue distributions of reduced density matrices. Commun. Math. Phys. 332, 1-52 (2014).

16 Gisin, N. Hidden quantum nonlocality revealed by local filters. Phys. Lett. A. 210, 151-156 (1996).

17 Verstraete, F., Dehaene, J. \& DeMoor, B. Local filtering operations on two qubits. Phys. Rev. A. 64, 010101 (2001).

18 Wang, Z.-W. et al. Experimental entanglement distillation of two-qubit mixed states under local operations. Phys. Rev. Lett. 96, 220505 (2006).

19 Bai, Y.-K. \& Wang, Z. D. Multipartite entanglement in four-qubit cluster-class states. Phys. Rev. A. 77, 032313 (2008).

20 Campbell, S., Tame, M. S. \& Paternostro, M. Characterizing multipartite symmetric dicke states under the effects of noise. New J. Phys. 11, 073039 (2009).

21 Bastin, T. et al. Operational determination of multiqubit entanglement classes via tuning of local operations. Phys. Rev. Lett. 102, 053601 (2009).

$22 \mathrm{Lu}, \mathrm{D}$. et al. Tomography is necessary for universal entanglement detection with single-copy observables. arXiv preprint arXiv:1511.00581 (2015). 
23 Qi, B. et al. Quantum state tomography via linear regression estimation. Sci. Rep 3, 3496 (2013).

24 Kwiat, P. G. et al. New high-intensity source of polarization-entangled photon pairs. Phys. Rev. Lett. 75, 4337-4341 (1995).

25 Zhang, C. et al. Experimental greenberger-horne-zeilinger-type six-photon quantum nonlocality. Phys. Rev. Lett. 115, 260402 (2015).

26 Pan, J.-W., Daniell, M., Gasparoni, S., Weihs, G. \& Zeilinger, A. Experimental demonstration of four-photon entanglement and high-fidelity teleportation. Phys. Rev. Lett. 86, 4435-4338 (2001).
This work is licensed under a Creative Commons Attribution 4.0 International License. The images or other third party material in this article are included in the article's Creative Commons license, unless indicated otherwise in the credit line; if the material is not included under the Creative Commons license, users will need to obtain permission from the license holder to reproduce the material. To view a copy of this license, visit http://creativecommons.org/licenses/by/ $4.0 /$

(c) The Author(s) 2017

Supplementary Information accompanies the paper on the npj Quantum Information website (doi:10.1038/s41534-017-0007-5). 